\title{
Light vector dark matter with scalar mediator and muon $g-2$ anomaly
}

\author{
Karim Ghorbani॰ \\ Physics Department, Faculty of Sciences, Arak University, Arak 38156-8-8349, Iran
}

(Received 31 August 2021; accepted 17 November 2021; published 8 December 2021)

\begin{abstract}
We study a model with a vector dark matter (DM) candidate interacting with the standard model (SM) charged leptons through a scalar portal. The dark matter candidate acquires mass when the complex scalar breaks an Abelian gauge symmetry spontaneously. The scalar interacts with the SM charged leptons through a dimension- 6 operator. The scalar mediator induces elastic scattering of dark matter with electrons at tree level and also DM-nucleon interaction when the effects from scalar-Higgs mixing are taken into account. Given the recent results from Xenon1T upper bounds on DM-electron elastic scattering cross sections where the strongest sensitivity lies in the range $\sim \mathcal{O}(1) \mathrm{GeV}$, we find the viable space in the parameter space respecting constraints from the observed relic density, direct detection, muon $\left(g_{\mu}-2\right)$ anomaly, $e^{+} e^{-}$colliders, electron beam-dump experiments and astrophysical observables. It is shown that the current upper bounds of Xenon1T on DM-electron interactions are partially sensitive to the regions in the viable parameter space which is already excluded by the electron beam-dump experiment, Orsay. We also find that there are viable DM particles with masses $\sim \mathcal{O}(1) \mathrm{GeV}$ evading the direct detection but standing well above the neutrino floor. Almost the same viable regions are found when we apply the direct detection upper limits on the DM-proton spin-independent cross section.
\end{abstract}

DOI: $10.1103 /$ PhysRevD.104.115008

\section{INTRODUCTION}

The nature of dark matter remains an unresolved problem, and the solution might reside at the intersection of cosmology and particle physics. The gravitational evidence for dark matter (DM) from cosmological observables is beyond doubt, but its particle nature is still hypothetical [1,2]. Weakly interacting massive particles (WIMPs) have been vastly studied as candidates for dark matter [3-5]. The mass of the WIMPy dark matter can be as light as that for axions [6] or it may emerge at the TeV scale $[7,8]$. The thermal production of DM in the early Universe, known as the freeze-out process [9], is a natural paradigm in cosmology resembling the same mechanism which has made very successful predictions for light element abundance and the cosmic microwave background. The mass of the dark matter particle and its interaction type are key ingredients in searching for its direct detection (DD). Interactions with velocity suppressed or momentum suppressed DM-nucleon scattering cross section are instances where DM may evade detection in direct and collider searches [10]. A direct search for DM candidates with masses of around $10 \mathrm{GeV}$ up to hundred $\mathrm{GeV}$ having

Published by the American Physical Society under the terms of the Creative Commons Attribution 4.0 International license. Further distribution of this work must maintain attribution to the author(s) and the published article's title, journal citation, and DOI. Funded by SCOAP .
DM-nucleon interactions has been a dedicated strategy in underground DD experiments [11-13]. In fact, we do not know if WIMPs should necessarily interact with the atomic nuclei. At any rate, if DM interacts with nucleons, it might be in the mass range in which the current DD experiments cannot exclude it. This in turn advocates the absence of DM-nucleon elastic scattering in current DD experiments.

One possible avenue in direct searches for dark matter is that DM might interact exclusively with the SM leptons and possibly have suppressed interactions with nucleons. The focus here is on WIMP candidates with masses in the range $\lesssim 10 \mathrm{GeV}$ communicating with the SM leptons by exchanging light scalar mediators. This type of interaction for DM receives stringent constraints from astrophysical and cosmological observations [14-17]. Additionally, searches beyond the SM in rare kaon decays $[18,19]$, $e^{+} e^{-}$colliders [20-22], beam-dump experiments [23-27], and muon anomalous magnetic moments (MAMMs) $[28,29]$ are highly motivated probes of light dark matter with a leptophilic scalar mediator. Moreover, we apply the newest results from Xenon1T [30], which probe DM-electron scattering for DM masses in the range (0.03-10) GeV.

The standard model (SM) prediction for the muon magnetic moment reads $a_{\mu}^{\mathrm{SM}}=(116591810 \pm 43) \times 10^{-11}$, where contributions from QED [31,32], QCD, or lattice QCD [33-48] and electroweak interactions [49,50] are taken into account with the highest precision. The first measurement indicating a deviation from the SM prediction 
was found by Brookhaven National Laboratory (BNL), $a_{\mu}^{\mathrm{BNL}}=(116592089 \pm 63) \times 10^{-11}$ [28]. The newest measurement which confirmed the deviation was announced by the Fermi National Laboratory (FNAL) with improved statistics, $a_{\mu}^{\mathrm{FNAL}}=(116592040 \pm 54) \times 10^{-11} \quad$ [29]. To explain the deviation, a large number of investigations applying various models beyond the SM are performed. Among them there are models introducing DM candidates interacting with the SM leptons via leptophilic scalar [5161], via a generic scalar mediator [62-64], and through vector mediator [65-68], with emphasis on the MAMMs.

This work examines a dark matter scenario in which the DM candidate is a vector gauge boson in an Abelian scalar gauge theory. The gauge boson acquires mass when the symmetry is broken spontaneously. Thus, the mass of the gauge boson is confined by the gauge coupling and the vacuum expectation value of the new scalar. On the other side, the scalar mediates the force between DM and the SM charged leptons. In this work the scalar interaction with the SM leptons is induced by dimension-6 operators. The models with a scalar mediator motivated by an effective field theory with dimension-5 operators are studied in [69-71]. The main purpose of this work is twofold. We would first like to see if DM candidates and appropriate scalar mediators can be found to explain the newest muon magnetic moment anomaly and, at the same time, satisfy other constraints from indirect searches. Finally, we investigate to find out whether the most strongest upper limits on DM-electron and DM-proton scattering cross section from Xenon1T are sensitive to the remaining viable parameter space.

The structure of the paper is as follows. In Sec. II the DM model is presented and the effective operators of dimension 6 are motivated by introducing a UV complete model. A discussion of the evaluation of the DM abundance is given in Sec. III. A couple of different terrestrial and astrophysical constraints are introduced in Sec. IV. Our final results are shown in Sec. V after imposing the upper bounds from DD experiments. We finish with a conclusion.

\section{MODEL}

The model we consider here contains a complex scalar field gauged under a $\mathrm{U}^{\prime}(1)$ symmetry with the Lagrangian

$$
\mathcal{L}_{\mathrm{DM}}=\left(D_{\mu} \phi\right)\left(D^{\mu} \phi\right)^{*}-m^{2} \phi \phi^{*}-\frac{1}{4} F^{\prime \mu \nu} F_{\mu \nu}^{\prime},
$$

where $D_{\mu}=\partial_{\mu}-i g_{\mathrm{v}} V_{\mu}$. The $\mathrm{U}^{\prime}(1)$ gauge symmetry is broken when the complex scalar field gets a nonzero vacuum expectation value, $v_{s}$. The scalar field can be parametrized as $\phi=\frac{1}{\sqrt{2}}\left(s+v_{s}\right) \exp \left(-i \pi / v_{s}\right)$. Here $s$ and $\pi$ are real scalar fields. The Goldstone boson is "eaten" by the longitudinal component of the gauge field giving a mass to the gauge boson as $M_{V}=g_{\mathrm{v}} v_{s}$.
In addition, one may consider a type of low energy effective interaction for the complex scalar $\phi$ in the form of a dimension-6 operator as $\sim \frac{1}{\Lambda_{l}^{2}}|\phi|^{2} \bar{L} H l_{R}$. Here $H$ is the SM Higgs doublet, $L$ is the SM left-handed lepton doublet, $l_{R}$ is the right-handed SM lepton, and $\Lambda_{l}$ is an appropriate energy scale for lepton $l$. In principle the dimension-6 operators including the SM quarks like $\frac{1}{\Lambda_{Q}^{2}}|\phi|^{2} \bar{Q} H^{\dagger} u_{R}$ and $\frac{1}{\Lambda_{Q}^{2}}|\phi|^{2} \bar{Q} H d_{R}$ are allowed by the symmetry. These interactions induce a large contribution to the DM-nucleon elastic scattering leading to the exclusion of the entire parameter space by the current direct detection bounds. Through a UV complete model we will propose a leptonspecific scenario in which only the leptonic operator is important.

Here we discuss a possible UV completion of the abovementioned effective interactions. To this end, we introduce a heavy new Higgs doublet, $\Phi$, with appropriate quantum numbers. The new doublet in general can have interactions with all the SM fermions. In this work we are interested in the so-called lepton-specific models in which the new doublet interacts only with the SM leptons. This type of interaction for the new doublet is motivated in two-Higgs doublet models [72-74]. We consider the following UV model:

$\mathcal{L}_{\mathrm{UV}}=y_{e} \Phi \bar{L}_{e} e_{R}+y_{\mu} \Phi \bar{L}_{\mu} \mu_{R}+y_{\tau} \Phi \bar{L}_{\tau} \tau_{R}+\kappa \Phi^{\dagger} H|\phi|^{2}+$ H.c.,

where $H$ is the SM Higgs doublet. In the limit in which the mass of the new doublet is heavy, integrating out the heavy doublet will lead us to the dimension-6 effective operator introduced earlier, i.e., $\sim \frac{1}{\Lambda_{l}^{2}}|\phi|^{2} \bar{L} H l_{R}$.

Therefore, if we assume that the new scalar interacts with the SM particles only through the leptonic operator, then it couples to the SM charged lepton currents effectively as

$$
\mathcal{L}_{\text {eff }}=\alpha_{l} s l^{+} l^{-},
$$

where $l=e, \mu, \tau$, and $\alpha_{l}$ is the corresponding effective coupling constant. The effective couplings of the scalar to leptons are parametrized to be mass-hierarchical couplings, $\alpha_{l}=\frac{m_{l}}{v_{s}} c_{l}$, which is intriguing phenomenologically. The cutoff scale $\Lambda_{l}$ is obtained as $\Lambda_{l}^{2} \sim v_{h} v_{s} / \alpha_{l}$, where $v_{h}$ is the vacuum expectation value of the SM Higgs. Following the same line of reasoning in [69], in the above effective Lagrangian we expect the two-loop contribution to the muon anomalous magnetic moment $\left(\Delta a_{\mu}\right)^{2 \text { loop }}$ and the one-loop contribution to the muon anomalous magnetic moment $\left(\Delta a_{\mu}\right)^{\text {lloop }}$, satisfying the relation $\left(\Delta a_{\mu}\right)^{2 \text { loop }} /\left(\Delta a_{\mu}\right)^{\text {lloop }} \sim \Lambda_{\mu}^{2} /\left(8 \pi^{2} v_{h}^{2}\right)$. To have a smaller two-loop contribution than one-loop contribution, we should have $\Lambda_{\mu}<2 \sqrt{2} \pi v_{h} \sim 2 \mathrm{TeV}$. 
TABLE I. The decay width $\Gamma\left(h \rightarrow s \tau^{+} \tau^{-}\right)$. The relevant effective coupling $\alpha_{\tau}=10^{-1}$.

\begin{tabular}{lccccccr}
\hline \hline$m_{s}(\mathrm{GeV})$ & $10^{-3}$ & 0.1 & 1 & 5 & 10 & 50 & 100 \\
\hline$\Gamma\left(10^{-5} \mathrm{GeV}\right)$ & 6.68 & 5.76 & 5.16 & 4.5 & 3.90 & 0.675 & 0.002 .49 \\
\hline \hline
\end{tabular}

The interaction Lagrangian which is relevant in this work includes these terms,

$\mathcal{L}_{\text {int }}=g_{\mathrm{v}}^{2} v_{s} s V_{\mu} V^{\mu}+\frac{1}{2} g_{\mathrm{v}}^{2} s^{2} V_{\mu} V^{\mu}+\alpha_{l} s l^{+} l^{-}+\frac{\alpha_{l}}{v_{h}} s h l^{+} l^{-}$.

The dark gauge boson $V$ is identified as our vector dark matter candidate. In the rest of the paper we shall use $m_{V}$ and $m_{\text {DM }}$ exchangeably. Moreover, we may consider another interaction term in the Lagrangian as $\sim \frac{1}{4} \lambda|\phi|^{2} H^{\dagger} H$, which can arise from the potential part of the UV model. We will justify below that, in order to respect bounds from the invisible Higgs decay, the coupling $\lambda$ should be negligible. This interaction is interesting here because it causes mixing between the singlet scalar and the SM Higgs, which can then lead to the invisible Higgs decay via the interaction $\sim\left(g_{v}^{2} v_{s} \sin \theta\right) h V_{\mu} V^{\mu}$. The mixing angle $\theta$ which diagonalizes the scalar mass matrix satisfies the relation $\sin 2 \theta=2 \lambda v_{s} v_{h} /\left(m_{h}^{2}-m_{s}^{2}\right)$. The SM Higgs invisible decay width in the decay process $h \rightarrow V V$ is given by the formula

$\Gamma_{\text {inv }}=\frac{g_{v}^{2} v_{s}^{2} m_{h}^{3} \sin ^{2} \theta}{16 \pi m_{V}^{4}}\left(1-4 x^{2}+12 x^{4}\right)\left(1-4 x^{2}\right)^{1 / 2}$,

where $x=m_{V} / m_{h}$. The observed upper limit at $95 \%$ confidence level on the branching ratio of the invisible Higgs decay is $\sim 0.19$ [75]. Depending on the region of parameter space that we explore in this work, it is found that if the mixing angle $\theta$ lies in the range $\lesssim 8 \times 10^{-4}$, then the respective regions evade bounds from invisible Higgs decay.

The last term in Eq. (4) opens up the possibility of a new decay channel for the SM Higgs. The Higgs particle can then decay to a scalar $s$ in the process $h \rightarrow s \bar{f} f$, where $f$ stands for the SM leptons. In the following we present some results for the decay width of $h \rightarrow s \tau^{+} \tau^{-}$in terms of the scalar mass. We picked out this decay channel because it has the largest decay width. The chosen value $\alpha_{\tau}=10^{-1}$ is large enough to find the upmost contribution to the Higgs total decay width. Since the decay width is proportional to $\alpha_{\tau}^{2}$, it is easy to estimate the decay width for other values of $\alpha_{\tau}$. To compute numerically the decay width $\Gamma\left(h \rightarrow s \tau^{+} \tau^{-}\right)$ the code CalcHEP [76] is employed. Our results for the decay width is presented in Table I. We can estimate that the total decay width, $\Gamma(h \rightarrow s \bar{f} f)$, for the scalar mass of interest in this work is of order $\sim 10^{-5} \mathrm{GeV}$. The total decay width of the SM Higgs is $3.2_{-2.2}^{+2.8} \mathrm{MeV}$ [77]. In conclusion, the total decay width $\Gamma(h \rightarrow s \bar{f} f)$ is about 2 orders of magnitude smaller than the Higgs total decay width, and therefore the measured Higgs decay width will not put any constraints on the relevant parameters.

\section{CONSTRAINTS FROM WMAP/PLANCK OBSERVATION}

With no evidence of $100 \mathrm{GeV} \mathrm{DM}$ in direct detection searches thus far, the interest has pushed toward low mass DM or light DM with $\sim \mathrm{GeV}$ DM particles. In this work we adopt thermal production of light DM particles through the so-called freeze-out mechanism, which sounds natural and is regarded as a standard mechanism for a thermal relic. During this thermal process DM annihilates to the SM particles (visibles) or other particles (secluded sector), and the reverse processes take place. The annihilation rate is in competition with the expansion rate of the Universe in the early Universe. There is a special temperature called the freeze-out temperature, $T_{f}$, or the decoupling temperature around which the DM particles get out of equilibrium and its density remains constant thereafter. The stronger the DM interaction with the SM particles, the longer it takes for DM particles to freeze out. The dark matter relic density is a function of the thermally averaged annihilation cross section, $\langle\sigma v\rangle$, as $\Omega h^{2} \propto\langle\sigma v\rangle^{-1}$. The observed value of the dark matter density is $\Omega h^{2} \approx 0.12$ $[16,17]$. The theoretical value for the DM relic density in the model parameter space is obtained by solving the relevant Boltzmann equation numerically applying micrOMEGAs [78].

Initially, we would like to find viable regions in the parameter space with DM masses in the range $10^{-3} \mathrm{GeV}<m_{\mathrm{DM}}<10 \mathrm{GeV}$, which gives rise to a relic abundance consistent with the observed value provided by WMAP [17] and Planck [16]. When thermal WIMPs have $s$-wave $2 \rightarrow 2$ annihilation to visible final states, the observed DM density puts a lower limit on the WIMP mass, $m \gtrsim 20 \mathrm{GeV}$ [79]. However, this is not the case when WIMPs also annihilate to a secluded dark sector. There are two possible DM annihilation channels for the DM particles in our model, namely, annihilation to a pair of dark scalars and annihilation to a pair of the SM charged leptons. In fact, we stay in a region of parameter space that $2 \rightarrow 2$ annihilation processes are dominant. DM particles can annihilate as $V V \rightarrow s s$ via $t$ and $u$ channels with the exchange of a vector boson and annihilation for a pair of dark scalars through a contact interaction if $m_{\mathrm{DM}}>m_{s}$. In addition, the $s$-channel DM annihilation, $V V \rightarrow s \rightarrow e^{+} e^{-}, \mu^{+} \mu^{-}, \tau^{+} \tau^{-}$, will be accessible when they are kinematically allowed. When we consider the 
<smiles>[3H]C([3H])C([3H])[3H]</smiles>
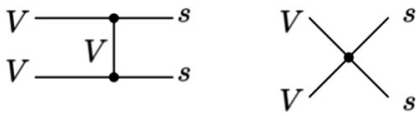

FIG. 1. Feynman diagrams for DM annihilation with dominant contributions.

scalar-Higgs mixing processes, another annihilation channel, $V V \rightarrow h \rightarrow f^{+} f^{-}$, becomes possible. However, since its annihilation cross section is proportional to $\sin ^{2} \theta$ and $\theta$ is restricted to quite small values, this effect has a very small contribution to the DM relic density. The relevant Feynman diagrams for the DM annihilation processes with dominant contributions are depicted in Fig. 1. The analytical formulas for the DM annihilation cross sections are given in the Appendix. The analytical results are confirmed after we implement our model in the code CalcHEP [76].

The parameter space that we scan over lies in the following intervals: $10^{-3} \mathrm{GeV}<m_{s}<100 \mathrm{GeV}, 0<g_{\mathrm{v}}<1$, $0<c_{e}=c_{\mu}=c_{\tau}<1$, and $1 \mathrm{GeV}<v_{s}<300 \mathrm{GeV}$. Remember that $m_{V}=m_{\mathrm{DM}}=g_{\mathrm{v}} v_{s}$ and $\alpha_{l}=\left(\frac{m_{l}}{v_{s}}\right) c_{l}$. In our scan the number of samplings is $10^{7}$. In each sampling,

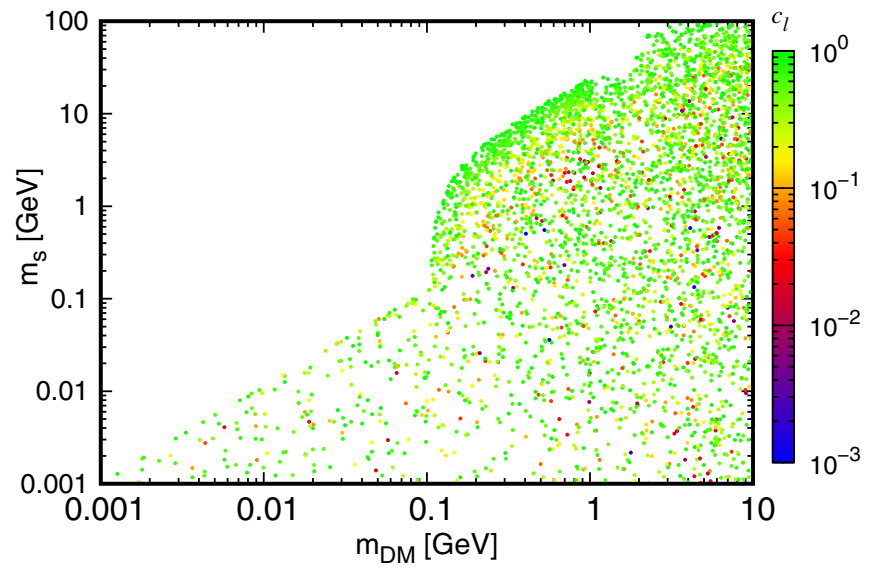

only when the computed relic density is consistent with the observed DM relic density can we keep the sampled free parameters. After finding the viable values for the parameters $c_{l}, g_{\mathrm{v}}, v_{s}, m_{s}$, and $m_{\mathrm{DM}}$, we present in the $m_{\mathrm{DM}}-m_{s}$ plane the resulting values for $c_{l}, g_{\mathrm{v}}$, and $v_{s}$, respectively, in three plots in Fig. 2. It is evident from the results shown in Fig. 2 that a larger DM mass toward $10 \mathrm{GeV}$ requires a larger mass for the scalar up to about $100 \mathrm{GeV}$.

\section{VARIOUS CONSTRAINTS ON SCALAR-MUON COUPLING}

In this section we discuss several types of constraints that might affect the viable parameter space.

(I) Muon anomalous magnetic moment.-The precise measurement of the muon anomalous magnetic moment, $a_{\mu}$, has been under intense scrutiny for a long time; for a review on this subject, see [80]. This quantity is defined as $a_{\mu}=\frac{g_{\mu}-2}{2}$, where $g_{\mu}$ is the wellknown gyromagnetic ratio for muon. At tree level in perturbation theory the quantity $g_{\mu}$ reads $g_{\mu}=2$. The SM radiative corrections include loop contributions from QED, QCD, and weak interactions.

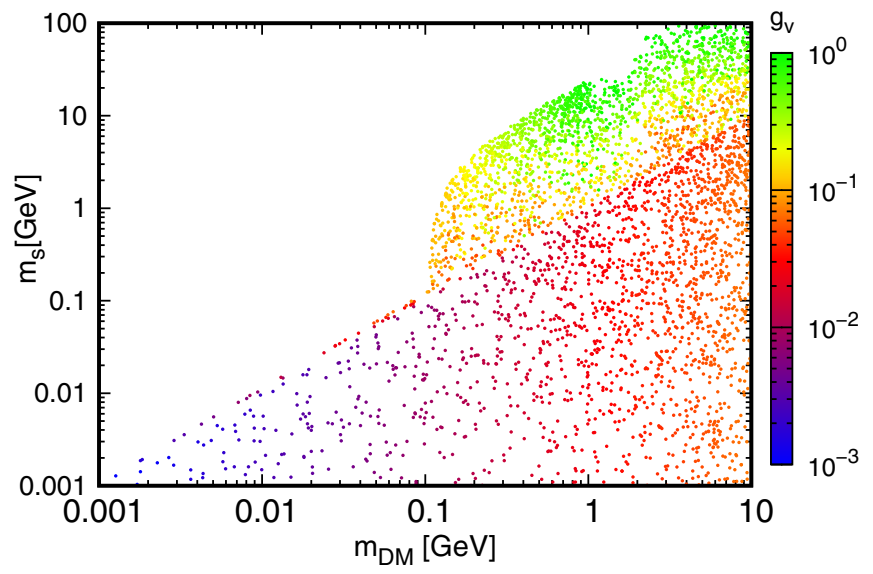

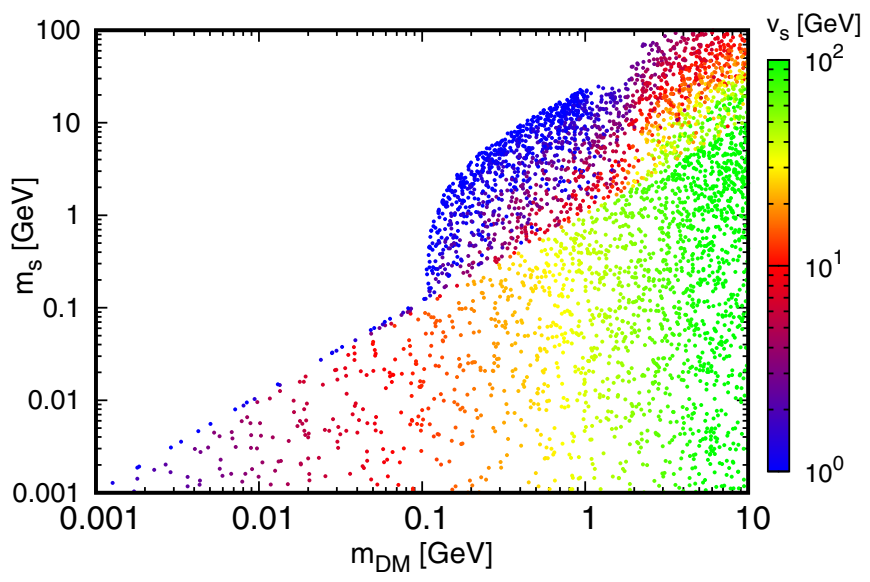

FIG. 2. Application of the constraints on the relic density from WMAP/Planck only. In the plane $m_{\mathrm{DM}}-m_{s}$, viable ranges for the parameters $m_{\mathrm{DM}}, m_{s}, c_{l}, g_{\mathrm{v}}$, and $v_{s}$ are shown in the three plots. 
The theoretical prediction of muon magnetic moment in the SM suffers mainly from the uncertainties in the hadronic vacuum polarization and the hadronic light-by-light scattering. A sizable deviation $\Delta a_{\mu}$ observed in past experiments at BNL [28] is considered a footprint of a probable new physics when one takes into account the controllable uncertainties from the theoretical side. Newly updated data from the muon $g-2$ experiment at FNAL not only supports the long-standing discrepancy but also provides results with improved statistics [29]. The new result comes along with a significance of about $4.2 \sigma$ and indicates a positive excess over the SM prediction. An updated experimental world average gives $\Delta a_{\mu}=a_{\mu}^{\exp }-a_{\mu}^{\mathrm{SM}}=(2.51 \pm 0.59) \times 10^{-9}$.

As a new physics effect, the scalar mediator in the present model will contribute to the muon anomalous magnetic moment at loop level and leads to the following correction:

$$
\begin{aligned}
\Delta a_{\mu}^{\mathrm{NP}}= & \frac{\alpha_{\mu}^{2}}{8 \pi^{2}} \int_{0}^{1} \frac{(1-z)^{2}(1+z)}{(1-z)^{2}+b^{2} z} d z \\
= & \frac{\alpha_{\mu}^{2}}{8 \pi^{2}}\left[\frac { 1 } { 2 } \left(-2 b^{2}+\left(b^{2}-3\right) b^{2} \log \left(b^{2}\right)\right.\right. \\
& \left.-2 \sqrt{b^{2}-4}\left(b^{2}-1\right) b \tanh ^{-1}\left(\frac{b^{2}-2}{b \sqrt{b^{2}-4}}\right)+3\right) \\
& \left.+\frac{b\left(b^{4}-5 b^{2}+4\right) \tanh ^{-1}\left(\frac{b}{\sqrt{b^{2}-4}}\right)}{\sqrt{b^{2}-4}}\right]
\end{aligned}
$$

where $b=\frac{m_{s}}{m_{\mu}}$. The new data on the muon magnetic moment deviation put stringent constraints on the scalar-muon coupling and the scalar mass.

(II) $e^{+} e^{-}$annihilation in colliders. - In $e^{+} e^{-}$colliders the production of the new scalar is possible through the process $e^{+} e^{-} \rightarrow \mu^{+} \mu^{-} s$. The scalar will subsequently decay to $\mu^{+} \mu^{-}$and therefore there are four muons in the final state. The BABAR experiment conducted a search in this channel and found the strongest upper limits on the effective coupling, $\alpha_{\mu}$, for $m_{s}>2 m_{\mu}$ [20]. For scalar masses with $m_{s}<2 m_{\mu}$, the Belle II experiment found constraints in a search for scalar production in the same channel but with the subsequent decay $s \rightarrow$ Invisible [21]. Moreover, the BABAR experiment has found constraints on a leptophilic scalar $\left(\Phi_{L}\right)$ decaying predominantly to leptons [22]. The limits constrain the scalar coupling for scalar masses up to $\sim 7 \mathrm{GeV}$.

(III) Beam-dump experiments.-Proton and electron beam-dump experiments are suitable probes in the search for new physics at low energy. In particular, a secondary muon beam originating from the original beam may radiate a dark scalar and the scalar can

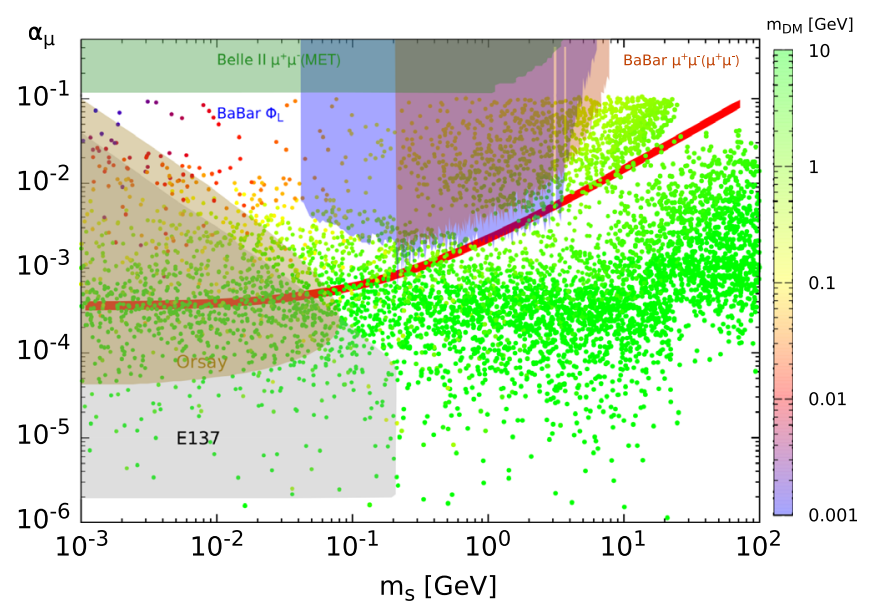

FIG. 3. Points in color show the viable space respecting the observed DM abundance in the $\alpha_{\mu}-m_{s}$ plane. Constraints from $e^{+} e^{-}$colliders and electron beam-dump experiments are imposed. The allowed region from muon anomalous magnetic moment is also shown as a red band.

subsequently decay into the SM leptons. Therefore, it is possible to search for the scalar-lepton coupling in these experiments. We apply exclusion limits on the scalar-muon coupling from two electron beamdump experiments, Orsay [23] and E137 [24].

(IV) Meson decays.- Since the scalar mediator $s$ in our model is leptophilic, the meson decays as $B \rightarrow K s$ and $K \rightarrow \pi s$ are not possible and there are no constraints in this regard.

(V) Supernova cooling.-Stellar cooling processes such as supernova cooling are types of probes which are sensitive to scalar-muon coupling for scalar masses below $\sim 1 \mathrm{MeV}$ [81]. In this work our interest is mainly in scalar masses $\gtrsim 1 \mathrm{MeV}$.

(VI) $B B N .-\mathrm{BBN}$ put constraints on the effective number of relativistic degrees of freedom beyond the SM particles with $\Delta N_{\text {eff }} \lesssim 0.2-0.6$ [82]. When our new particles have mass $\gtrsim \mathcal{O}(1) \mathrm{MeV}$, the parameter space is not sensitive to the BBN bounds [83].

Taking into account all the constraints mentioned in this section and that from the observed relic density, we show the viable region of the parameter space in Fig. 3. The supernova cooling constraints exclude the allowed region by the $g_{\mu}-2$ anomaly for scalar masses smaller than $\sim 1 \mathrm{MeV}$, while the BABAR [in the process $\left.e^{+} e^{-} \rightarrow \mu^{+} \mu^{-}\left(\mu^{+} \mu^{-}\right)\right]$and Belle II upper limits do not overlap with the allowed region. However, the limits from $B A B A R$ (in the process $e^{+} e^{-} \rightarrow \tau^{+} \tau^{-} \Phi_{L}$ ) partially exclude the allowed region in the scalar mass range $\sim 1-4 \mathrm{GeV}$. It is also seen that in the remaining parameter space respecting the $g_{\mu}-2$ allowed region, the observed relic density and beam-dump experiments, DM mass varies in the range $\sim 0.1-10 \mathrm{GeV}$ and the scalar mass varies in the range $\sim 0.07-20 \mathrm{GeV}$. The strongest lower limits on the scalar mass belong to two electron beam-dump experiments 


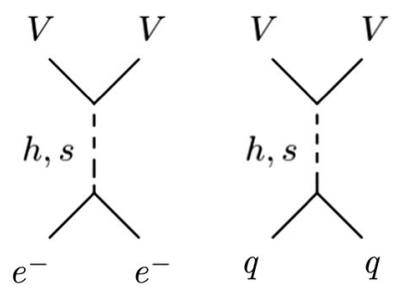

FIG. 4. Feynman diagrams for DM-electron and DM-quark elastic scattering at tree level.

where scalar masses smaller than $\sim 0.07 \mathrm{GeV}$ are excluded by the Orsay experiment.

\section{DIRECT DETECTION BOUNDS}

In our model spin-independent (SI) DM-nucleon interaction is present at tree level due to the scalar-Higgs mixing effects. In addition, DM-electron elastic scattering of the spin-independent type is feasible at tree level. In the following we ignore the loop suppressed DM-matter interactions. In Fig. 4 Feynman diagrams for the DMelectron scattering at tree level and also DM-quark scattering at tree level are depicted. We obtain a reference DM-electron direct detection cross section,

$$
\sigma^{e} \sim \frac{4}{3 \pi} \alpha_{e}^{2} g_{\mathrm{v}}^{2} \frac{\mu_{\mathrm{ve}}^{2}}{\left(m_{s}^{2}+\alpha^{2} m_{e}^{2}\right)^{2}},
$$

where the reduced mass of the DM electron is $\mu_{\mathrm{ve}}$ and the electron momentum transfer is typically set as $q \sim \alpha m_{e}$. The contribution of the diagram with the Higgs propagator to the DM-electron cross section is numerically negligible since the mixing angle, $\theta$, is very small. In the limit in which $m_{s} \gg \alpha m_{e}$, the DM form factor $F_{\mathrm{DM}} \sim 1$ [84]. To date, in direct detection experiments no evidence of DMelectron elastic scattering has been found. However, recently the experimental results from Xenon10 [84] and DarkSide-50 [85] set upper bounds on DM-electron for masses below $\sim 1 \mathrm{GeV}$ and Xenon1T [30] provides stringent bounds on the DM-electron cross section for DM masses in the range $\sim 0.03-10 \mathrm{GeV}$ [30]. On the other hand, the neutrino floor sets the lowest limits for the scattering cross section of dark matter with visible matter. We apply the latest result for the neutrino floor given in [86]. In this section we pick out points in the parameter space which respect all the relevant constraints discussed previously, including those from beam-dump experiments, the observed relic density. We also confine the parameter space to the regions allowed by the muon $\left(g_{\mu}-2\right)$ anomaly. The regions in the parameter space that we scan over are $0<g_{\mathrm{v}}<1, \quad 1 \mathrm{GeV}<v_{s}<300 \mathrm{GeV}, 0<c_{l}<1$, and $10^{-3} \mathrm{GeV}<m_{s}<10 \mathrm{GeV}$. For the DM mass we have $m_{V}=g_{\mathrm{v}} \times v_{s}$, and the relevant effective coupling here is $\alpha_{e}=\frac{m_{e}}{v_{s}} c_{e}$. The final result for the DM-electron elastic scattering cross section in terms of the DM mass and the scalar mass, $m_{s}$, is shown in the left panel of Fig. 5 and, in terms of the DM mass and the coupling, $\alpha_{e}$, is presented in the right panel of Fig. 5. The results indicate that Xenon1T, having the strongest limits among the DD experiments, is sensitive only to the region with the scalar mass which is already excluded by the electron beam-dump experiment, Orsay. However, there are regions with $m_{s} \gtrsim 0.07 \mathrm{GeV}$ and with dark matter mass in the range $1 \mathrm{GeV} \lesssim m_{\mathrm{DM}} \lesssim$ $10 \mathrm{GeV}$ which evade the current Xenon1T bounds and stand well above the neutrino floor.

The Xenon1T [30] and DarkSide-50 [85] collaborations provide bounds on the DM-nucleon cross section for DM masses below $10 \mathrm{GeV}$, as shown in Fig. 6. We apply the package microMEGAs to compute the DM-proton SI cross section in the parameter space in the same ranges that we discussed in the DM-electron case. Concerning the mixing angle, $\theta$, we always pick values to respect the invisible

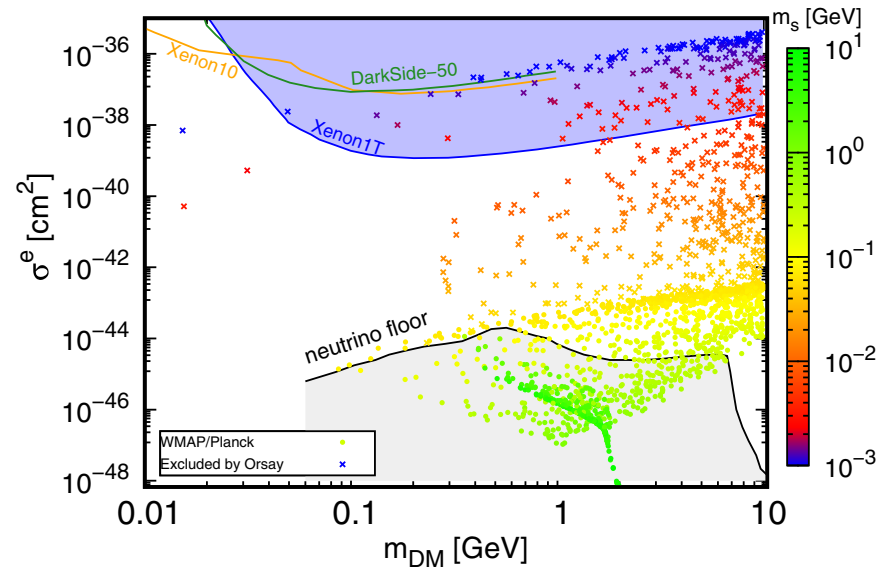

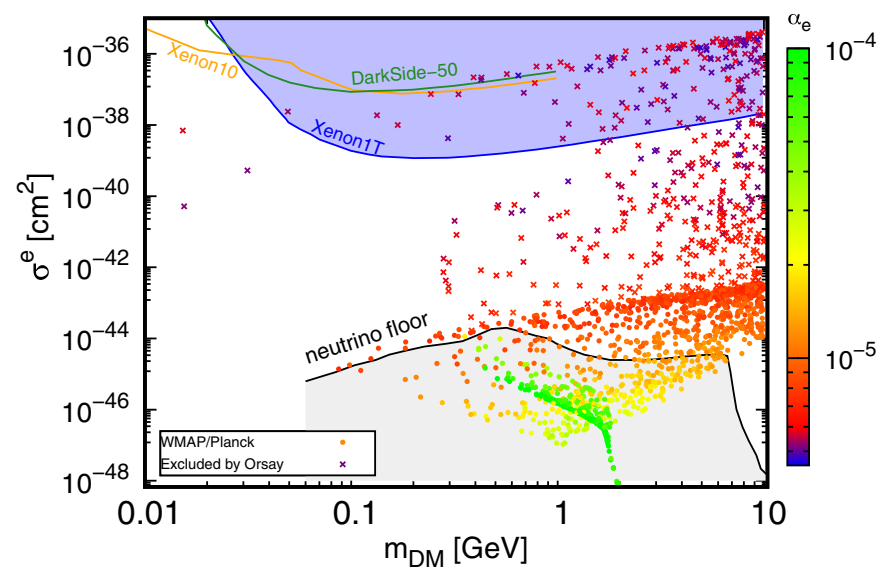

FIG. 5. We show regions in the parameter space which respect all the constraints discussed in the text and also points which are excluded by the electron beam-dump experiment, Orsay. All the points respect the allowed region by the muon $\left(g_{\mu}-2\right)$ anomaly. The upper bounds from direct detection experiments on the DM-electron elastic cross section are shown. The scalar mass, $m_{s}$ (left panel), and the coupling, $\alpha_{e}$ (right panel), are shown in the vertical color spectrum. The neutrino floor is shaded in gray. 


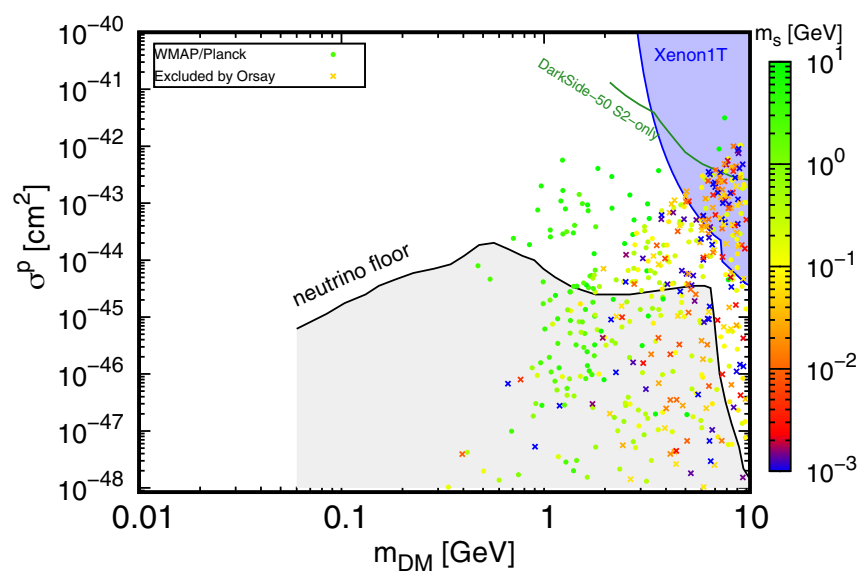

FIG. 6. Regions in the parameter space which respect all the constraints discussed in the text and points which are excluded by the electron beam-dump experiment, Orsay. All the points respect the allowed region by the muon $\left(g_{\mu}-2\right)$ anomaly. The upper bounds from direct detection experiments on the DM-proton elastic cross section are shown. The viable values for the scalar mass, $m_{s}$, are also shown. The neutrino floor is shaded in gray.

Higgs decay bounds. We show our results in Fig. 6 for points which respect all the restrictions and also points which are excluded by the Orsay beam-dump experiment. We find that there are viable DM candidates with masses $\sim 0.7-10 \mathrm{GeV}$ and scalar mass $m_{s} \sim 0.1-10 \mathrm{GeV}$ with a SI cross section well above the neutrino floor and the available DD bounds respected.

\section{CONCLUSION}

In light of the newest results from the muon magnetic moment anomaly $\left(g_{\mu}-2\right)$ and DM-matter elastic scattering upper bounds from Xenon-1T, we exemplified a vector DM model with a scalar mediator which is coupled to the SM charged leptons via dimension- 6 operators. We introduced a UV complete model to illustrate the types of dimension- 6 operators used in our study. From a phenomenological point of view, we confined the dark matter mass to the range $10^{-3} \mathrm{GeV}<m_{\mathrm{DM}}<10 \mathrm{GeV}$ and the scalar masses in the range $10^{-3} \mathrm{GeV}<m_{s}<100 \mathrm{GeV}$.

In the first part of the analysis we imposed constraints from the observed DM density, muon anomalous magnetic moment, supernova cooling, $e^{+} e^{-}$colliders, and electron beam-dump experiments. The viable range for the scalar mass is then obtained as $0.07 \mathrm{GeV} \lesssim m_{s} \lesssim 20 \mathrm{GeV}$ and for the DM mass as $0.1 \mathrm{GeV} \lesssim m_{\mathrm{DM}} \lesssim 10 \mathrm{GeV}$. Next we computed the DM-electron elastic scattering cross section. We then applied the upper limits from the DD experiments, Xenon100, DarkSide, and Xenon1T and found that the strongest bound from Xenon1T excludes scalar masses with $m_{s} \lesssim 3 \mathrm{MeV}$ for DM masses $0.1 \mathrm{GeV} \lesssim m_{\mathrm{DM}} \lesssim 10 \mathrm{GeV}$. Since we had already found that the electron beam-dump experiment, Orsay, excluded scalar masses with $m_{s}<0.07 \mathrm{GeV}$, we can conclude that the current DD experiments via DM-electron interaction have an almost 2 order of magnitude weaker sensitivity reach for the scalar mass than the electron beam-dump experiments. Given that the neutrino floor is increasing in the region with a DM mass smaller than $10 \mathrm{GeV}$, we are still able to find $\mathrm{DM}$ candidates of $\mathcal{O}(1) \mathrm{GeV}$ with direct detection cross section about 2 orders of magnitude above the neutrino floor.

Moreover, considering the DM-nucleon interaction for a DM mass below $10 \mathrm{GeV}$, viable regions are found that have not yet been explored by DD experiments, and further improvements on the experimental bounds in this mass range would be essential to further constrain or exclude the dark matter models.

\section{ACKNOWLEDGMENTS}

The author would like to thank Dr. Parsa Ghorbani for useful discussions.

\section{APPENDIX: ANNIHILATION CROSS SECTIONS}

Here we present the formulas for the DM annihilation cross section times the relative velocity. The annihilation cross section for the $s$-channel annihilation process $V V \rightarrow l^{+} l^{-}$, with $l=e, \mu, \tau$, is first obtained as

$\sigma_{\text {anni }} v_{\text {rel }}\left(V V \rightarrow l^{+} l^{-}\right)=\frac{2 \alpha_{l}^{2} v_{s}^{2} g_{\mathrm{v}}^{4}}{9 \pi^{2}} \frac{\left(1-4 m_{l}^{2} / s\right)^{3 / 2}}{\left(s-m_{s}^{2}\right)^{2}}$

And then we find the DM annihilation cross section with a pair of singlet scalars in the final state,

$$
\begin{aligned}
\sigma_{\text {anni }} v_{\text {rel }}(V V \rightarrow s s) \\
=\frac{\sqrt{1-4 m_{s}^{2} / s}}{16 \pi^{2} s} \int d \Omega\left[\frac{64}{9} v_{s}^{4} g_{\mathrm{v}}^{8}\left(\frac{1}{t-m_{V}^{2}}+\frac{1}{u-m_{V}^{2}}\right)^{2}\right. \\
\left.\quad-\frac{64}{9} v_{s}^{2} g_{\mathrm{v}}^{6}\left(\frac{1}{t-m_{V}^{2}}+\frac{1}{u-m_{V}^{2}}\right)+\frac{8}{9} g_{\mathrm{v}}^{4}\right]
\end{aligned}
$$

where $s, t$, and $u$ are the relevant Mandelstam variables. The relative velocity of the incoming DM particles is denoted by $v_{\text {rel }}$. 
[1] J. L. Feng, Dark matter candidates from particle physics and methods of detection, Annu. Rev. Astron. Astrophys. 48, 495 (2010).

[2] G. Bertone, D. Hooper, and J. Silk, Particle dark matter: Evidence, candidates and constraints, Phys. Rep. 405, 279 (2005).

[3] G. Steigman and M. S. Turner, Cosmological constraints on the properties of weakly interacting massive particles, Nucl. Phys. B253, 375 (1985).

[4] G. Arcadi, M. Dutra, P. Ghosh, M. Lindner, Y. Mambrini, M. Pierre, S. Profumo, and F. S. Queiroz, The waning of the WIMP? A review of models, searches, and constraints, Eur. Phys. J. C 78, 203 (2018).

[5] L. Bergström, Nonbaryonic dark matter: Observational evidence and detection methods, Rep. Prog. Phys. 63, 793 (2000).

[6] M. Kawasaki and K. Nakayama, Axions: Theory and cosmological role, Annu. Rev. Nucl. Part. Sci. 63, 69 (2013).

[7] K. Griest and M. Kamionkowski, Unitarity Limits on the Mass and Radius of Dark-Matter Particles, Phys. Rev. Lett. 64, 615 (1990).

[8] K. Blum, Y. Cui, and M. Kamionkowski, An ultimate target for dark matter searches, Phys. Rev. D 92, 023528 (2015).

[9] G. Steigman, B. Dasgupta, and J. F. Beacom, Precise relic WIMP abundance and its impact on searches for dark matter annihilation, Phys. Rev. D 86, 023506 (2012).

[10] E. D. Nobile, Appendiciario: A hands-on manual on the theory of direct Dark Matter detection, arXiv:2104.12785.

[11] D. S. Akerib et al. (LUX Collaboration), Results from a Search for Dark Matter in the Complete LUX Exposure, Phys. Rev. Lett. 118, 021303 (2017).

[12] E. Aprile et al. (XENON Collaboration), First Dark Matter Search Results from the XENON1T Experiment, Phys. Rev. Lett. 119, 181301 (2017).

[13] R. Agnese et al. (SuperCDMS Collaboration), Results from the Super Cryogenic Dark Matter Search Experiment at Soudan, Phys. Rev. Lett. 120, 061802 (2018).

[14] R. H. Cyburt, B. D. Fields, K. A. Olive, and T.-H. Yeh, Big bang nucleosynthesis: Present status, Rev. Mod. Phys. 88, 015004 (2016).

[15] G. G. Raffelt, Stars as Laboratories for Fundamental Physics: The Astrophysics of Neutrinos, Axions, and Other Weakly Interacting Particles (University of Chicago Press, Chicago, 1996), p. 5.

[16] P. A. R. Ade et al. (Planck Collaboration), Planck 2015 results. XIII. Cosmological parameters, Astron. Astrophys. 594, A13 (2016).

[17] G. Hinshaw et al. (WMAP Collaboration), Nine-year Wilkinson Microwave Anisotropy Probe (WMAP) observations: Cosmological parameter results, Astrophys. J. Suppl. Ser. 208, 19 (2013).

[18] E. Cortina Gil et al. (NA62 Collaboration), Search for $\mathrm{K}^{+}$ decays to a muon and invisible particles, Phys. Lett. B 816, 136259 (2021).

[19] G. Krnjaic, G. Marques-Tavares, D. Redigolo, and K. Tobioka, Probing Muonphilic Force Carriers and Dark Matter at Kaon Factories, Phys. Rev. Lett. 124, 041802 (2020).
[20] J. P. Lees et al. (BABAR Collaboration), Search for a muonic dark force at BABAR, Phys. Rev. D 94, 011102 (2016).

[21] I. Adachi et al. (Belle-II Collaboration), Search for an Invisibly Decaying $Z^{\prime}$ Boson at Belle II in $e^{+} e^{-} \rightarrow$ $\mu^{+} \mu^{-}\left(e^{ \pm} \mu^{\mp}\right)$ plus Missing Energy Final States, Phys. Rev. Lett. 124, 141801 (2020).

[22] J. P. Lees et al. (BABAR Collaboration), Search for a Dark Leptophilic Scalar in $e^{+} e^{-}$Collisions, Phys. Rev. Lett. 125, 181801 (2020).

[23] M. Davier and H. Nguyen Ngoc, An unambiguous search for a light Higgs boson, Phys. Lett. B 229, 150 (1989).

[24] J. D. Bjorken, S. Ecklund, W. R. Nelson, A. Abashian, C. Church, B. Lu, L.W. Mo, T. A. Nunamaker, and P. Rassmann, Search for neutral metastable penetrating particles produced in the SLAC beam dump, Phys. Rev. D 38, 3375 (1988).

[25] S. N. Gninenko, N. V. Krasnikov, and V. A. Matveev, Muon $g-2$ and searches for a new leptophobic sub-GeV dark boson in a missing-energy experiment at CERN, Phys. Rev. D 91, 095015 (2015).

[26] C.-Y. Chen, M. Pospelov, and Y.-M. Zhong, Muon beam experiments to probe the dark sector, Phys. Rev. D 95, 115005 (2017).

[27] L. Marsicano, M. Battaglieri, A. Celentano, R. De Vita, and Y.-M. Zhong, Probing leptophilic dark sectors at electron beam-dump facilities, Phys. Rev. D 98, 115022 (2018).

[28] G. W. Bennett et al. (Muon $g-2$ Collaboration), Final report of the muon E821 anomalous magnetic moment measurement at BNL, Phys. Rev. D 73, 072003 (2006).

[29] B. Abi et al. (Muon $g-2$ Collaboration), Measurement of the Positive Muon Anomalous Magnetic Moment to 0.46 ppm, Phys. Rev. Lett. 126, 141801 (2021).

[30] E. Aprile et al. (XENON Collaboration), Light Dark Matter Search with Ionization Signals in XENON1T, Phys. Rev. Lett. 123, 251801 (2019).

[31] T. Aoyama, M. Hayakawa, T. Kinoshita, and M. Nio, Complete Tenth-Order QED Contribution to the Muon $g-2$, Phys. Rev. Lett. 109, 111808 (2012).

[32] T. Aoyama, T. Kinoshita, and M. Nio, Theory of the anomalous magnetic moment of the electron, Atoms 7, 28 (2019).

[33] M. Davier, A. Hoecker, B. Malaescu, and Z. Zhang, Reevaluation of the hadronic vacuum polarisation contributions to the standard model predictions of the muon $g-2$ and $\alpha\left(m_{Z}^{2}\right)$ using newest hadronic cross-section data, Eur. Phys. J. C 77, 827 (2017).

[34] A. Keshavarzi, D. Nomura, and T. Teubner, Muon $g-2$ and $\alpha\left(M_{Z}^{2}\right)$ : A new data-based analysis, Phys. Rev. D 97, 114025 (2018).

[35] G. Colangelo, M. Hoferichter, and P. Stoffer, Two-pion contribution to hadronic vacuum polarization, J. High Energy Phys. 02 (2019) 006.

[36] M. Hoferichter, B.-L. Hoid, and B. Kubis, Three-pion contribution to hadronic vacuum polarization, J. High Energy Phys. 08 (2019) 137.

[37] A. Keshavarzi, D. Nomura, and T. Teubner, $g-2$ of charged leptons, $\alpha\left(M_{Z}^{2}\right)$, and the hyperfine splitting of muonium, Phys. Rev. D 101, 014029 (2020). 
[38] A. Kurz, T. Liu, P. Marquard, and M. Steinhauser, Hadronic contribution to the muon anomalous magnetic moment to next-to-next-to-leading order, Phys. Lett. B 734, 144 (2014).

[39] M. Davier, A. Hoecker, B. Malaescu, and Z. Zhang, A new evaluation of the hadronic vacuum polarisation contributions to the muon anomalous magnetic moment and to $\boldsymbol{\alpha}\left(m_{Z}^{2}\right)$, Eur. Phys. J. C 80, 241 (2020); Erratum, Eur. Phys. J. C 80, 410 (2020).

[40] S. Borsanyi et al., Leading hadronic contribution to the muon magnetic moment from lattice QCD, Nature (London) 593, 51 (2021).

[41] K. Melnikov and A. Vainshtein, Hadronic light-by-light scattering contribution to the muon anomalous magnetic moment revisited, Phys. Rev. D 70, 113006 (2004).

[42] P. Masjuan and P. Sanchez-Puertas, Pseudoscalar-pole contribution to the $\left(g_{\mu}-2\right)$ : A rational approach, Phys. Rev. D 95, 054026 (2017).

[43] M. Hoferichter, B.-L. Hoid, B. Kubis, S. Leupold, and S. P. Schneider, Dispersion relation for hadronic light-by-light scattering: Pion pole, J. High Energy Phys. 10 (2018) 141.

[44] A. Gérardin, H. B. Meyer, and A. Nyffeler, Lattice calculation of the pion transition form factor with $N_{f}=2+1$ Wilson quarks, Phys. Rev. D 100, 034520 (2019).

[45] V. Pauk and M. Vanderhaeghen, Single meson contributions to the muon's anomalous magnetic moment, Eur. Phys. J. C 74, 3008 (2014).

[46] I. Danilkin and M. Vanderhaeghen, Light-by-light scattering sum rules in light of new data, Phys. Rev. D 95, 014019 (2017).

[47] P. Roig and P. Sanchez-Puertas, Axial-vector exchange contribution to the hadronic light-by-light piece of the muon anomalous magnetic moment, Phys. Rev. D 101, 074019 (2020).

[48] T. Blum, N. Christ, M. Hayakawa, T. Izubuchi, L. Jin, C. Jung, and C. Lehner, Hadronic Light-by-Light Scattering Contribution to the Muon Anomalous Magnetic Moment from Lattice QCD, Phys. Rev. Lett. 124, 132002 (2020).

[49] A. Czarnecki, W. J. Marciano, and A. Vainshtein, Refinements in electroweak contributions to the muon anomalous magnetic moment, Phys. Rev. D 67, 073006 (2003); Erratum, Phys. Rev. D 73, 119901 (2006).

[50] C. Gnendiger, D. Stöckinger, and H. Stöckinger-Kim, The electroweak contributions to $(g-2)_{\mu}$ after the Higgs boson mass measurement, Phys. Rev. D 88, 053005 (2013).

[51] P. Agrawal, Z. Chacko, and C. B. Verhaaren, Leptophilic dark matter and the anomalous magnetic moment of the muon, J. High Energy Phys. 08 (2014) 147.

[52] C. Boehm, X. Chu, J.-L. Kuo, and J. Pradler, Scalar dark matter candidates revisited, Phys. Rev. D 103, 075005 (2021).

[53] R. Garani and J. Heeck, Dark matter interactions with muons in neutron stars, Phys. Rev. D 100, 035039 (2019).

[54] S. Yaser Ayazi and A. Mohamadnejad, DAMPE excess from leptophilic vector dark matter: A model-independent approach, J. Phys. G 47, 095003 (2020).

[55] J. Liu, X.-P. Wang, and K.-P. Xie, Searching for lepton portal dark matter with colliders and gravitational waves, J. High Energy Phys. 06 (2021) 149.

[56] Y. Bai and J. Berger, Muon $g-2$ in lepton portal dark matter, arXiv:2104.03301.
[57] S.-F. Ge, X.-D. Ma, and P. Pasquini, Probing the dark axion portal with muon anomalous magnetic moment, Eur. Phys. J. C 81, 787 (2021).

[58] S.-I. Horigome, T. Katayose, S. Matsumoto, and I. Saha, Leptophilic fermion WIMP: Role of future lepton colliders, Phys. Rev. D 104, 055001 (2021).

[59] J. C. Eung and T. Mondal, Leptophilic bosons and muon $g-2$ at lepton colliders, J. High Energy Phys. 07 (2021) 044.

[60] D. Borah, M. Dutta, S. Mahapatra, and N. Sahu, Muon $(g-2)$ and XENON1T excess with boosted dark matter in $L_{\mu}-L_{\tau}$ model, Phys. Lett. B 820, 136577 (2021).

[61] W. Yin, Muon $g-2$ anomaly in anomaly mediation, J. High Energy Phys. 06 (2021) 029.

[62] P. Athron, C. Balázs, D. H. Jacob, W. Kotlarski, D. Stöckinger, and H. Stöckinger-Kim, New physics explanations of $a_{\mu}$ in light of the FNAL muon $g-2$ measurement, J. High Energy Phys. 09 (2021) 080.

[63] G. Arcadi, L. Calibbi, M. Fedele, and F. Mescia, Muon $g-2$ and $B$ Anomalies from Dark Matter, Phys. Rev. Lett. 127, 061802 (2021).

[64] B. Zhu and X. Liu, Probing the flavor-specific scalar mediator for the muon $(g-2)$ deviation, the proton radius puzzle and the light dark matter production, arXiv:2104 .03238

[65] N. F. Bell, Y. Cai, R. K. Leane, and A. D. Medina, Leptophilic dark matter with $Z^{\prime}$ interactions, Phys. Rev. D 90, 035027 (2014).

[66] K. Ghorbani and P. H. Ghorbani, DAMPE electron-positron excess in leptophilic $Z^{\prime}$ model, J. High Energy Phys. 05 (2018) 125.

[67] P. Athron, C. Balazs, A. Fowlie, and Y. Zhang, Modelindependent analysis of the DAMPE excess, J. High Energy Phys. 02 (2018) 121.

[68] G. Arcadi, A. S. De Jesus, T. B. De Melo, F. S. Queiroz, and Y.S. Villamizar, A 2 HDM for the $g-2$ and dark matter, arXiv:2104.04456.

[69] B. Batell, A. Freitas, A. Ismail, and D. Mckeen, Flavorspecific scalar mediators, Phys. Rev. D 98, 055026 (2018).

[70] B. Batell, N. Lange, D. McKeen, M. Pospelov, and A. Ritz, Muon anomalous magnetic moment through the leptonic Higgs portal, Phys. Rev. D 95, 075003 (2017).

[71] C.-Y. Chen, H. Davoudiasl, W. J. Marciano, and C. Zhang, Implications of a light dark Higgs solution to the $g_{\mu}-2$ discrepancy, Phys. Rev. D 93, 035006 (2016).

[72] S. Su and B. Thomas, The LHC discovery potential of a leptophilic Higgs, Phys. Rev. D 79, 095014 (2009).

[73] G. C. Branco, P. M. Ferreira, L. Lavoura, M. N. Rebelo, M. Sher, and J. P. Silva, Theory and phenomenology of twoHiggs-doublet models, Phys. Rep. 516, 1 (2012).

[74] G. Marshall and M. Sher, The supersymmetric leptophilic Higgs model, Phys. Rev. D 83, 015005 (2011).

[75] A. M. Sirunyan et al. (CMS Collaboration), Search for invisible decays of a Higgs boson produced through vector boson fusion in proton-proton collisions at $\sqrt{s}=13 \mathrm{TeV}$, Phys. Lett. B 793, 520 (2019).

[76] A. Belyaev, N. D. Christensen, and A. Pukhov, CalcHEP3.4 for collider physics within and beyond the standard model, Comput. Phys. Commun. 184, 1729 (2013). 
[77] P. A. Zyla et al. (Particle Data Group), Review of particle physics, Prog. Theor. Exp. Phys. (2020) 083C01.

[78] G. Belanger, F. Boudjema, A. Pukhov, and A. Semenov, micromEGAs3: A program for calculating dark matter observables, Comput. Phys. Commun. 185, 960 (2014).

[79] R. K. Leane, T. R. Slatyer, J. F. Beacom, and K. C. Y. Ng, GeV-scale thermal WIMPs: Not even slightly ruled out, Phys. Rev. D 98, 023016 (2018).

[80] T. Aoyama et al., The anomalous magnetic moment of the muon in the standard model, Phys. Rep. 887, 1 (2020).

[81] R. Bollig, W. DeRocco, P. W. Graham, and H.-T. Janka, Muons in Supernovae: Implications for the Axion-Muon Coupling, Phys. Rev. Lett. 125, 051104 (2020).
[82] G. Mangano and P. D. Serpico, A robust upper limit on $N_{\text {eff }}$ from BBN, circa 2011, Phys. Lett. B 701, 296 (2011).

[83] M. Pospelov and J. Pradler, Big bang nucleosynthesis as a probe of new physics, Annu. Rev. Nucl. Part. Sci. 60, 539 (2010).

[84] R. Essig, J. Mardon, and T. Volansky, Direct detection of sub-GeV dark matter, Phys. Rev. D 85, 076007 (2012).

[85] P. Agnes et al. (DarkSide Collaboration), Constraints on Sub-GeV Dark-Matter-Electron Scattering from the DarkSide-50 Experiment, Phys. Rev. Lett. 121, 111303 (2018).

[86] J. Billard et al., Direct detection of dark matter: APPEC committee report, arXiv:2104.07634. 